\title{
Calcitonin inhibits production of immunoglobulins, rheumatoid factor and interleukin- 1 by mononuclear cells from patients with rheumatoid arthritis
}

\author{
Sumihisa Aida, Masako Okawa-Takatsuji, Shinichi Aotsuka, Koki Shimoji, \\ Ryuichi Yokohari
}

\begin{abstract}
Objectives-Elcatonin (eCT), an eel calcitonin derivative, is shown to considerably improve the clinical signs and symptoms, as well as laboratory data, in patients with rheumatoid arthritis (RA). The therapeutic efficacy of eCT, however, is reduced by preceding and/or concomitant use of corticosteroid. Thus the effects of eCT on the production of immunoglobulins, IgMRF and interleukin-1 (IL-1) by mononuclear cells (MNCs)/monocytes were studied, and compared among patients with RA that received three kinds of treatment and also normal volunteers (NV).

Methods-Ten patients with RA had been treated with a non-steroidal anti-inflammatory drug only (NSAID group), 11 with oral prednisolone (PSL group), and eight with intramuscular eCT (eCT group). MNCs/monocytes from these patients, and also 10 from the NV group, were collected and cultured. IgG, IgA, IgM, IgMRF, IL-1 $\alpha$ and $I L-1 \beta$ in the supernatants were measured by enzyme-linked immunosorbent assay (ELISA). In the NSAID, PSL and NV groups, eCT was added to the culture medium, and the effects of eCT on production of these substances were studied. Results-Baseline production of IgM, IL$1 \alpha$ and IL-1 $\beta$ by MNCs/monocytes in the eCT and NV groups was significantly lower than that in the NSAID group. Furthermore, addition of eCT to the culture medium significantly inhibited the productions of IgG, IgMRF, IL-1 $\alpha$ and IL-1 $\beta$ by MNCs/monocytes in the NSAID group, whereas production of neither IgG, IgA, IgM, IgMRF nor IL-1 by MNCs/monocytes in the PSL and NV groups was affected by eCT.

Conclusion-eCT may regulate immune responses through MNC/monocyte function in patients with RA. The present results support our proposal that $e C T$ is an effective agent for the treatment of RA.
\end{abstract}

(Ann Rheum Dis 1994; 53: 247-249)

Calcitonin (CT), a hormone produced in the thyroid gland, is widely known to inhibit bone resorption by osteoclasts, ${ }^{1-3}$ and has been used for treatment of osteoporosis. In patients with rheumatoid arthritis (RA), however, we have demonstrated that treatment with elcatonin (eCT), an eel CT derivative, considerably improved not only the clinical signs and symptoms but also decreased the erythrocyte sedimentation rate (ESR), and the serum levels of C-reactive protein (CRP), IgG, IgA, IgM and IgM-rheumatoid factor (IgMRF). Furthermore, the efficacy of eCT was suggested to be altered by preceding and/or concomitant use of corticosteroid. ${ }^{45}$ In this study we investigated the effects of eCT on production of $\operatorname{IgG}, \operatorname{Ig} A, \operatorname{IgM}, \operatorname{Ig} M R F$ by cultured mononuclear cells (MNC)s, as well as production of IL- $1 \alpha$ and IL- $1 \beta$ by monocytes.

\section{Patients and methods}

Twenty nine patients with $\mathrm{RA}^{6}$ and 10 normal volunteers were studied after obtaining their informed consent. Ten patients with RA, as controls, had been treated with a non-steroidal anti-inflammatory drug (NSAID) only, although the type of drug and its dose varied (NSAID group, nine women, one man; mean age, 61 years; mean stage [7], 1·7). Eleven patients with RA had been treated additionally with oral prednisolone, $2 \cdot 5-5.0 \mathrm{mg} /$ day (PSL group; nine women, two men; mean age, 55 years; mean stage, $2 \cdot 3$ ). Eight patients had been additionally treated with intramuscular eCT, 20 MRC units/week, for more than six months (eCT group; seven women, one man; mean age, 63 years; mean stage, 2.9). Ten normal volunteers (NV group; nine women, one man; mean age, 58 years) were used as controls. There were no significant differences in sex or stage (Chi square test), as well as age, ESR and CRP (one-way analysis of variance (ANOVA)) among the groups.

After separation by centrifugation on a Ficoll-Hypaque gradient ${ }^{8}$ and washing three times with Hank's balanced salt solution, peripheral blood MNCs from patients with RA were suspended in RPMI 1640 supplemented with $5 \%$ fetal calf serum (FCS). Monocytes in MNCs were isolated according to the method of Montazeri et $a l^{9}$ by allowing them to adhere to polystyrene tissue culture dishes coated with FCS. In the NSAID and PSL groups, eCT (Asahi Chemical Industries, Tokyo, Japan) at final concentrations of $0,1,10$ and $50 \mathrm{ng} / \mathrm{ml}$ 
was added to the culture medium. After incubation of the dishes at $37^{\circ} \mathrm{C}$ with $5 \% \mathrm{CO}_{2}$ for seven days for MNCs and for 20 hours for monocytes, IgG, IgA, IgM, IgMRF, IL- $1 \alpha$ and IL-1 $\beta$ in the supernatants were measured by enzyme-linked immunosorbent assay (ELISA) as described previously. ${ }^{10-12}$ Population and viability of $\mathrm{MNCs} / \mathrm{monocytes}$ was verified photomicroscopically before and after culture.

Results were expressed as median and quartile deviation, $\mathrm{Q}\left(\mathrm{Q}=\left(\mathrm{Q}_{3}-\mathrm{Q}_{1}\right) / 2\right)$, when non-parametric tests were used, and as mean and standard error of the mean(SEM) when the parametric test was used. Differences and

$\lg G$

$\lg A$
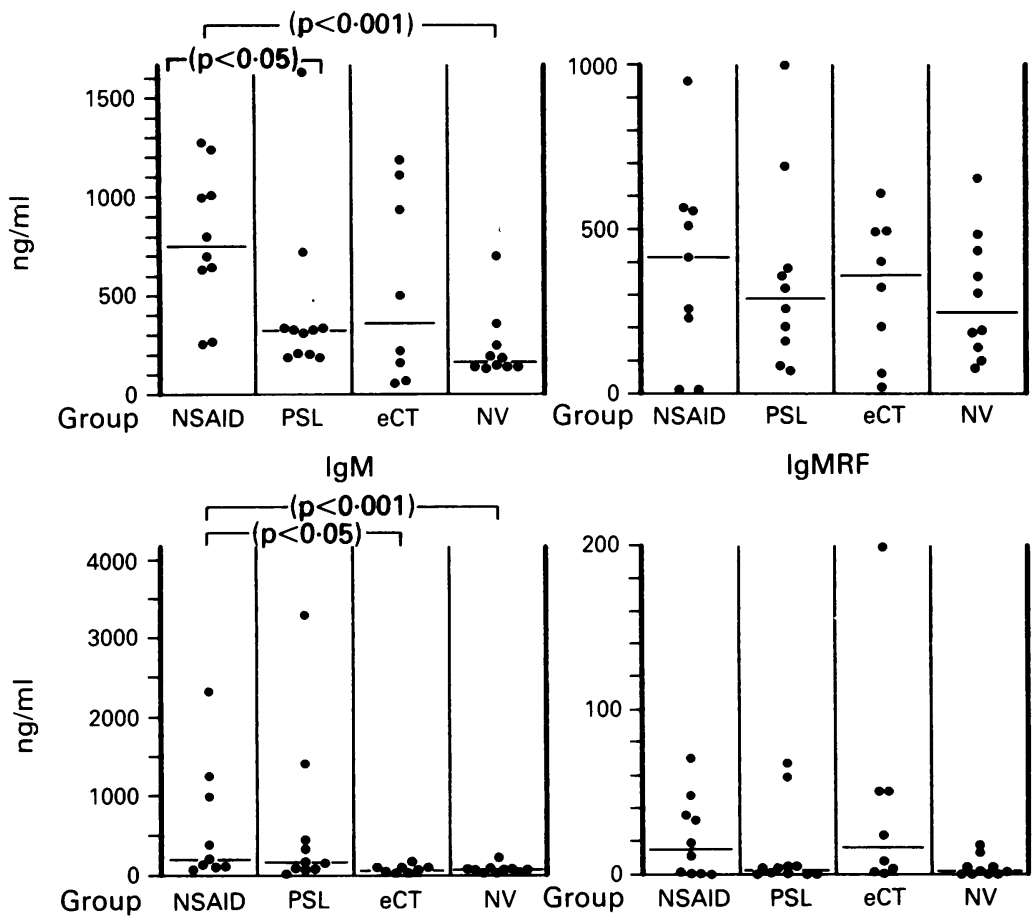

Figure 1 Baseline concentrations of $\operatorname{Ig} G, \operatorname{Ig} A, \operatorname{Ig} M$ and IgMRF produced by cultured mononuclear cells from patients with rheumatoid arthritis and normal volunteers. Baseline concentrations of IgM produced by MNCs/monocytes in the eCT group, as well as IgG and IgM in the NV group were significantly lower than those in the NSAID group. NSAID, previous treatment with non-steroidal anti-inflammatory drugs (NSAID)s; PSL, previous treatment with prednisolone (PSL); $e C T$, previous treatment with elcatonin (eCT), an eel calcitonin derivative. Horizontal lines represent the medians. Mann-Whitney U test was used.
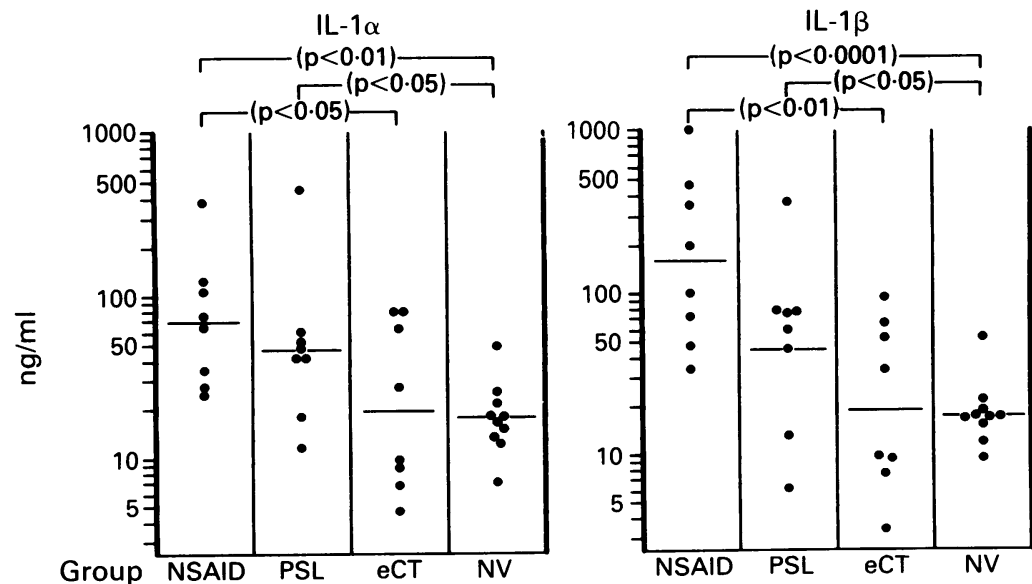

Figure 2 Baseline concentrations of $I L-1 \alpha$ and $I L-1 \beta$ produced by cultured monocytes from patients with rheumatoid arthritis and normal volunteers. Baseline concentrations of from patients with rheumatoid arthritis and normal volunteers. Baseline concentrations lower than those in the NSAID group. Horizontal lines represent the means. Student's t test was used. changes in immunoglobulins and IgMRF were analysed by the Mann-Whitney $U$ test and Friedman test, respectively. Differences and changes in IL-1, whose dispersion showed approximately a normal distribution on a logarithmic scale, were analysed by Student's $t$ test and two-way ANOVA respectively, after transformation into common logarithms. Differences at $\mathrm{p}<0.05$ were considered significant.

\section{Results}

Baseline concentrations (of medium without eCT application) of $\operatorname{IgM}(\mathrm{p}<0.05), \mathrm{IL}-1 \alpha$ $(\mathrm{p}<0.05)$ and IL-1 $\beta(\mathrm{p}<0.01)$ produced by MNCs/monocytes in the eCT group, as well as IgG $(p<0.001), \quad \operatorname{IgM}(p<0.001), \quad$ IL-1 $\alpha$ $(\mathrm{p}<0.01)$ and IL-1 $\beta(\mathrm{p}<0.0001)$ in the NV group were significantly lower than those of the NSAID group (Mann-Whitney U test for IgG and IgM, Student's $t$ test for IL- $1 \alpha$ and IL-1 $\beta$ ). In the PSL group, only IgG was significantly low compared with the NSAID group (figs 1, 2).

The addition of eCT to the culture medium of $\mathrm{MNCs} /$ monocytes from the NSAID group decreased the production of IgG $(p<0.05)$ and IgMRF ( $p<0.05)$, IL- $1 \alpha(p<0.001)$ and IL-1 $\beta(p<0.01)$ (Friedman test for IgG and IgMRF, two-way ANOVA for IL- $1 \alpha$ and IL$1 \beta$ ) in a dose-dependent manner (figs 3,4 ). In the PSL and NV groups, however, these suppressive effects of eCT on the production of immunoglobulins, IgMRF and cytokines by MNCs/monocytes were not significant (figs $3,4)$.

\section{Discussion}

The present results showed that in vivo treatment with eCT inhibits the production of IgM (fig 1 ), IL- $1 \alpha$ and IL-1 $\beta$ (fig 2 ) by MNCs/ monocytes obtained from patients with RA, and also that in vitro application of eCT inhibits the production of IgG, IgMRF (fig 3), IL- $1 \alpha$ and IL-1 $\beta$ (fig 4 ) by MNCs/monocytes obtained from patients with RA given NSAIDs only. Furthermore, these effects were not significant in the PSL and NV groups. The inhibitory effects of eCT on both immunoglobulin and IgMRF production were compatible with our clinical observations of patients with RA treated with eCT. ${ }^{45}$

It has been reported that $\mathrm{CT}$ receptors are present on human lymphocytes, ${ }^{13}{ }^{14}$ monocytes and macrophages ${ }^{15} 16$ as well as on osteoclasts. ${ }^{1-3}$ Binding of CT by these receptors has been shown to inhibit mitogenesis in thymocytes, ${ }^{17}$ and the accumulation of cyclic nucleotides in $\mathrm{MNCs}^{18}$ and monocytes or macrophages. ${ }^{15}$ Although one of the roles of CT is inhibition of bone resorption by osteoclasts, ${ }^{1-3}$ which are considered to be derived from monocytes, ${ }^{19}$ another possible associated action of CT might be suppression of immune responses.

The precise mechanism of this suppressive effect of eCT on MNCs/monocytes is still unclear at present, but multiple actions may be 

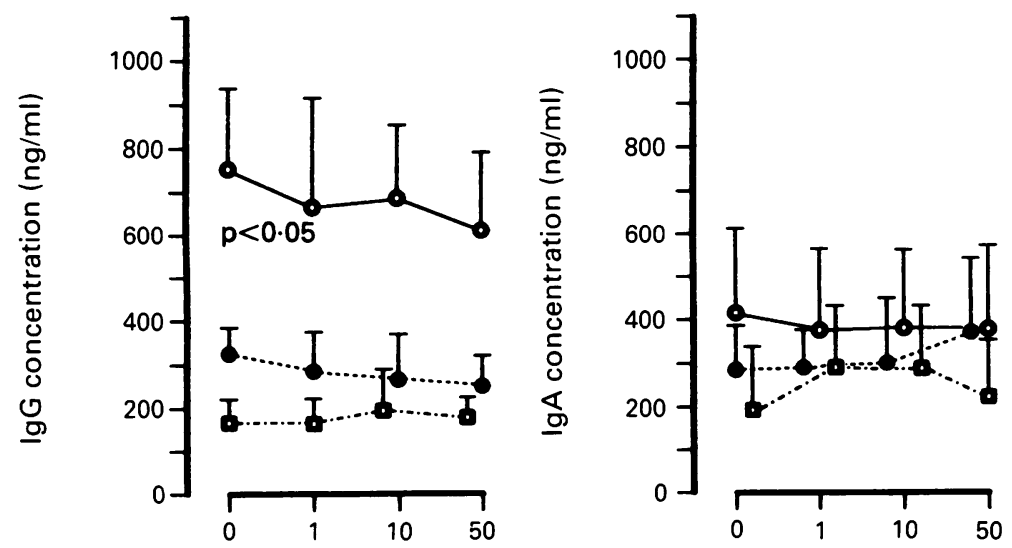

prednisolone. Furthermore, MNCs/monocytes from the NV group were not affected by eCT. This suggests that eCT allows a normal immune response to be retained but regulates the pathological immune response in RA. These findings agree with previous studies, which have demonstrated that treatment of RA with eCT decreases the serum levels of immunoglobulins and IgMRF without side-effects, and that preceding and/or concomitant use of corticosteroid negates these clinical effects. ${ }^{45}$ The present study therefore supports our proposal that $\mathrm{eCT}$ is an effective therapeutic agent for RA.
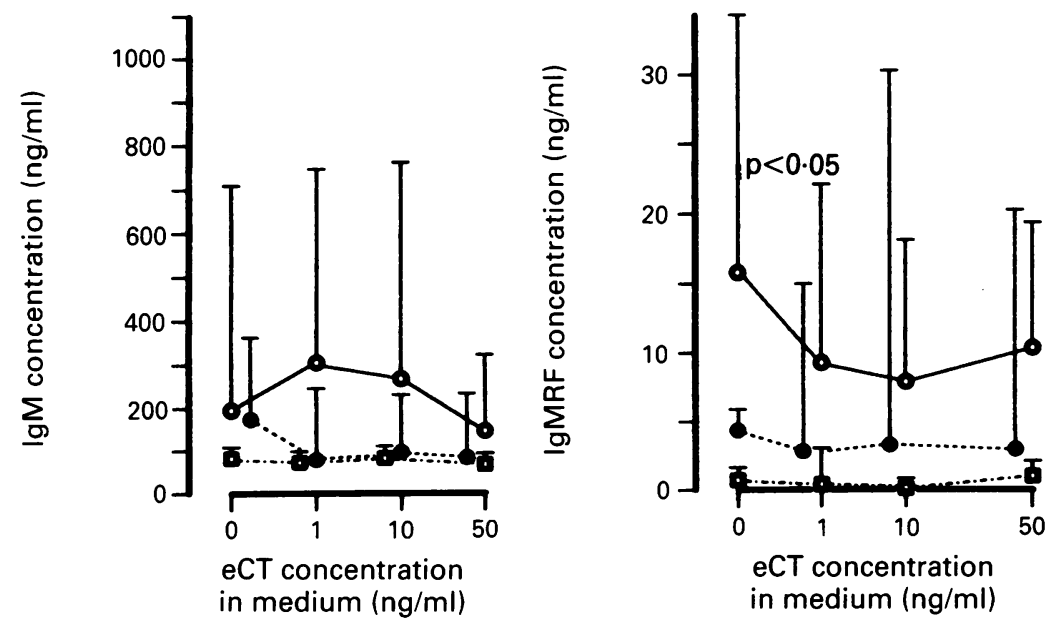

Figure 3 Effects of elcatonin (eCT) on production of $\operatorname{IgG}, \operatorname{Ig} A, \operatorname{IgM}$ and IgMRF by cultured mononuclear cells from patients with rheumatoid arthritis and normal volunteers. Addition of eCT to the incubation medium of monocytes from the NSAID group decreased the production of IgG and IgMRF. Symbols represent the medians and quartile deviations, $\left.Q\left(Q=Q_{3}-Q_{1}\right) / 2\right)$, where position (on or under the symbol) of each vertical bar indicates the direction of wider deviation; $0, N S A I D$ group $(n=10) ; \bullet, P S L$ group $(n=11) ; \square, N V$ group $(n=10)$. Friedman test was used.
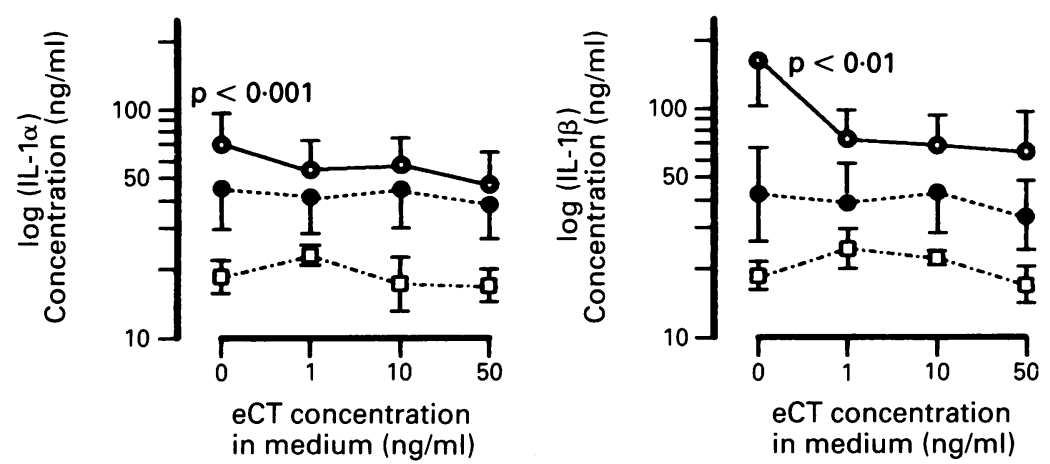

Figure 4 Effects of elcatonin (eCT) on production of IL-1 $\alpha$ and IL-1 $\beta$ by cultured monocytes from patients with rheumatoid arthritis and normal volunteers. Addition of $e C T$ to the culture medium of monocytes from the NSAID group decreased the production of IL$1 \alpha$ and IL-1 3 . Symbols represent the means and standard error of the mean (SEM); $O$, NSAID group $(n=8) ; \bullet, P S L$ group $(n=8) ; \square, N V$ group $(n=10)$. Two-way ANOVA was used.

involved: not only direct action but also indirect actions on MNCs/monocytes appear to be present. eCT inhibited IL- $\alpha$ and IL-1 $\beta$ production in this study, suggests that the suppressive effects on immunoglobulins were elicited indirectly via suppression of the synthesis of some cytokines, such as IL- $1 \alpha$ and IL-1 $\beta .{ }^{20} 21$

Our findings suggest strong regulatory effects of eCT on immune responses in RA, and interference with these effects by

1 Friedman J, Reisz G. Thyrocalcitonin: inhibitor of bone resorption in tissue culture. Science 1965; 150: 1465-7.

2 Aliapoulis M A, Goldhaber P, Munson P L. Thyrocalcitonin inhibition of bone resorption induced by parathyroid hormone in tissue culture. Science 1966; 151: 330-1.

3 Takamura $T$, Kanda $S$. The role of macrophages in osteoblast neogenesis in vivo: Quantitative analysis of osteoclasts and macrophages in endochondral bone development in rat treated with hydrocortisone and calcitonin. Metab Bone Dis E Rel Res 1983; 5: 139-143.

4 Aida S, Shimoji K. Effects of calcitonin on rheumatoid arthritis and the relation with corticosteroids. Pain Res 1990; 5: 85-89.

5 Aida S. Effects of eel calcitonin on rheumatoid arthritis. Ann Rheum Dis 1991; 50: 202-3.

6 Arnett F C, Edworthy S M, Bloch D A, et al. The American Rheumatism Association 1987. Revised criteria for the classification of rheumatoid arthritis. Arthritis Rheum 1988; 31: 315-24.

7 Steinblocker O, Traeger C H, Batterman R C. Therapeutic criteria in rheumatoid arthritis. $f A M A$ 1949; 140: 659-62.

8 Böyum A. Separation of leucocytes from blood and bone, marrow. Scand 7 Clin Lab Invest 1968; 21: 77-89.

9 Montazeri G, Chiorazzi N, Fu S M, Kunkel H G. Regulatory role of circulating monocytes in the differentiative and proliferative responses of human $B$ lymphocytes. Clin Immunol Immunopathol 1980; 16: lymphoc 10 .

10 Okawa-Takatsuji M, Aotsuka S, Uwatoko S, Yokohari R, Inagaki K. Monocyte-mediated suppression of rheumatoid factor production in normal subjects. Clin Immunol Immunopathol 1988; 46: 195-204.

11 Okawa-Takatsuji M, Aotsuka S, Uwatoko S, Uwatoko S, Sumiya $M$, Yokohari $R$. The $B$ cell repertoire in patients with systemic autoimmune diseases: analysis of EpsteinBarr virus (EBV)-inducible circulating precursors that produce autoantibodies against nuclear ribonucleoprotein (nRNP). Clin Exp Immunol 1992; 90: 415-21.

12 Aotsuka $S$, Nakamura $\mathrm{K}$, Nakano $\mathrm{T}$, et al. Production of intracellular and extracellular interleukin-1 $\alpha$ and interleukin- $1 \beta$ by peripheral blood monocytes from patients with connective tissue diseases. Ann Rheum Dis 1991; 50: 27-31.

13 Marx S J, Aurbach G D, Gavin J R, Buell D W. Calcitonin receptors on cultured human lymphocytes. $\mathcal{F}$ Biol Chem 1974; 249: 6812-16.

14 Body J J, Glibert F, Nejai S, Fernandez G, Van Langendonck $A$. Calcitonin receptors on circulating normal human lymphocytes. F Clin Endocrinol Metab 1990; 71: 675-81.

15 Popoff'S N, Coderre J A, Marks S C Jr, Stock J L. Calcitonin inhibits accumulation of cyclic AMP in stimulated peritoneal macrophages from normal rats but not from osteopetrotic (incisors-absent) littermates. Life Sci 1989; 45: 2007-13.

16 Sacerdote P, Bianchi M, Panerai A E. Human monocyte chemotactic activity of calcitonin and somatostatinrelated peptides: modulation by chronic peptide treatment. $\mathcal{F}$ Clin Endocrinol Metab 1990; 70: 141-8.

17 MacManus J P, Whitfield J F. Inhibition by thyrocalcitonin of the mitogenic actions of parathyroid hormone and cyclic adenosine $3^{\prime}, 5$ '-monophosphate on rat thymocytes. endocrinology 1970; 86: 934-9.

18 Stock J L, Coderre J A. Calcitonin and parathyroid hormone inhibit accumulation of cyclic AMP in stimulated human mononuclear cells. Biochem Biophys Res Commun 1982 109: $935-42$

19 Nijweide P J, Burger E H, Feyen J H. Cells of bone: proliferation, differentiation and hormonal regulation. Physiol Rev 1986; 66: 855-86.

20 Kirkham B. Interleukin-1, immune activation pathways and different mechanisms in osteoarthritis and rheumatoid arthritis. Ann Rheum Dis 1991; 50: 395-400.

21 Wahl S M. Cellular and molecular interactions in the induction of inflammation in rheumatic diseases. In: Kresina T F, ed. Monoclonal antibodies, cytokines, and arthritis. New York: Marcel Dekker Inc, 1991: 101-32. 